\title{
THE RISK OF SEVERE COVID-19 AND MORTALITY FROM COVID-19 IN PEOPLE LIVING WITH HIV COMPARED TO INDIVIDUALS WITHOUT HIV - A SYSTEMATIC REVIEW AND META-ANALYSIS OF 1268676 INDIVIDUALS
}

\section{NTRODUCTION}

HIV epidemic meets COVID-19 pandemic

- As of 2020 at least 38 million people were living with HIV globally, more than two-thirds of PLHIV being in SubSaharan Africa ${ }^{1}$.

- Since December 2020, the number of confirmed cases of COVID-19 has increased in Sub-Saharan Africa ${ }^{2}$, where health systems are ill equipped to treat surges in the need for hospitalization, mechanical ventilation and intensive care services associated with severe COVID-19.

- Identifying groups of people who are susceptible to severe COVID-19 and death from COVID-19 is a priority, as these groups may need additional protections and prioritization for vaccination against COVID-193,4

- There are indications that people living with HIV (PLHIV) may be one such vulnerable group due to persistent immune-suppression ${ }^{5}$.

- There is conflicting evidence about the risk of mortality and severe disease due to COVID-19 in people living with HIV (PLHIV).

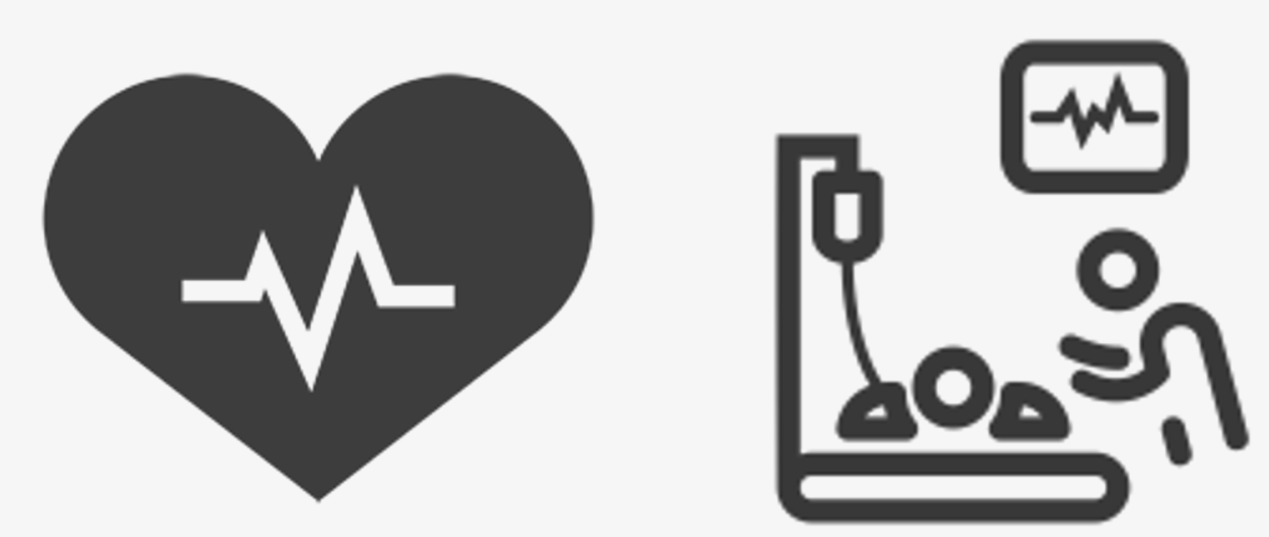

\section{AIM}

To compare mortality, hospitalization, and the need for intensive care services due to COVID-19 between PLHIV \& individuals without HIV based on data from the existing literature

\section{RESULTS}

\section{$1,268,676$}
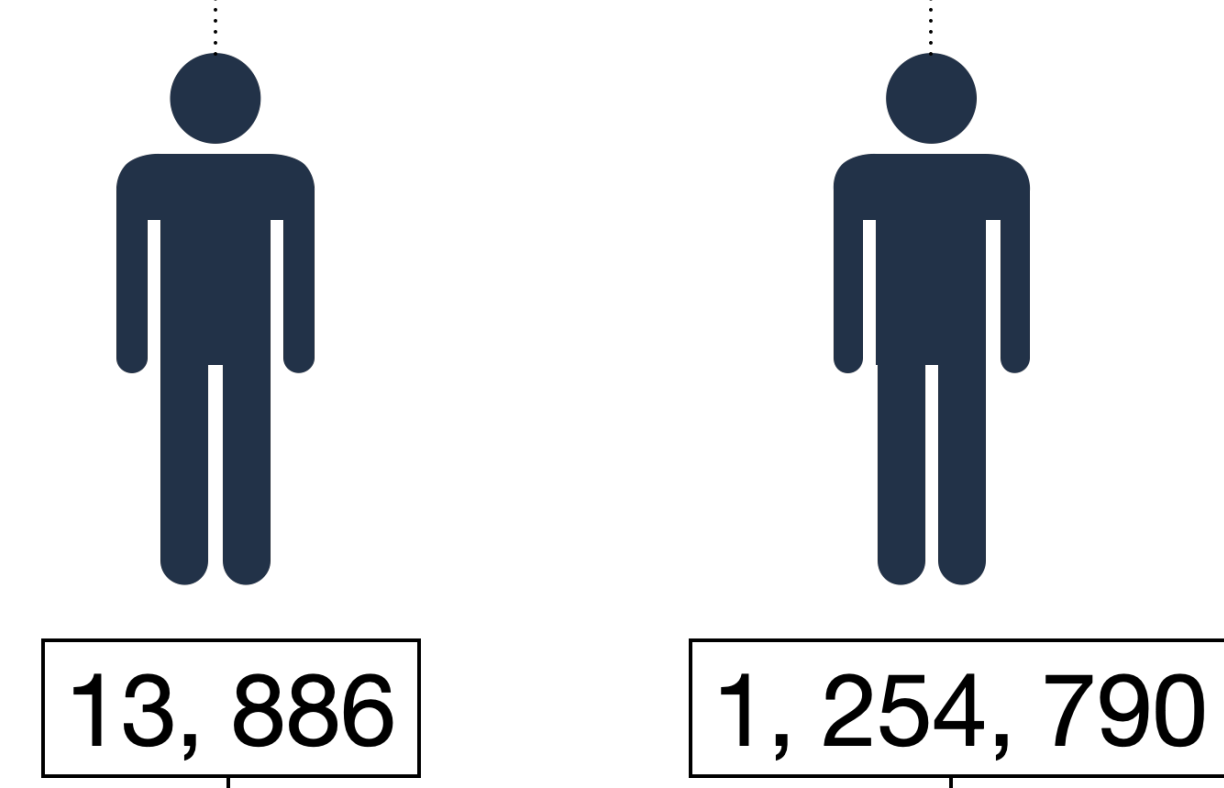

13,886

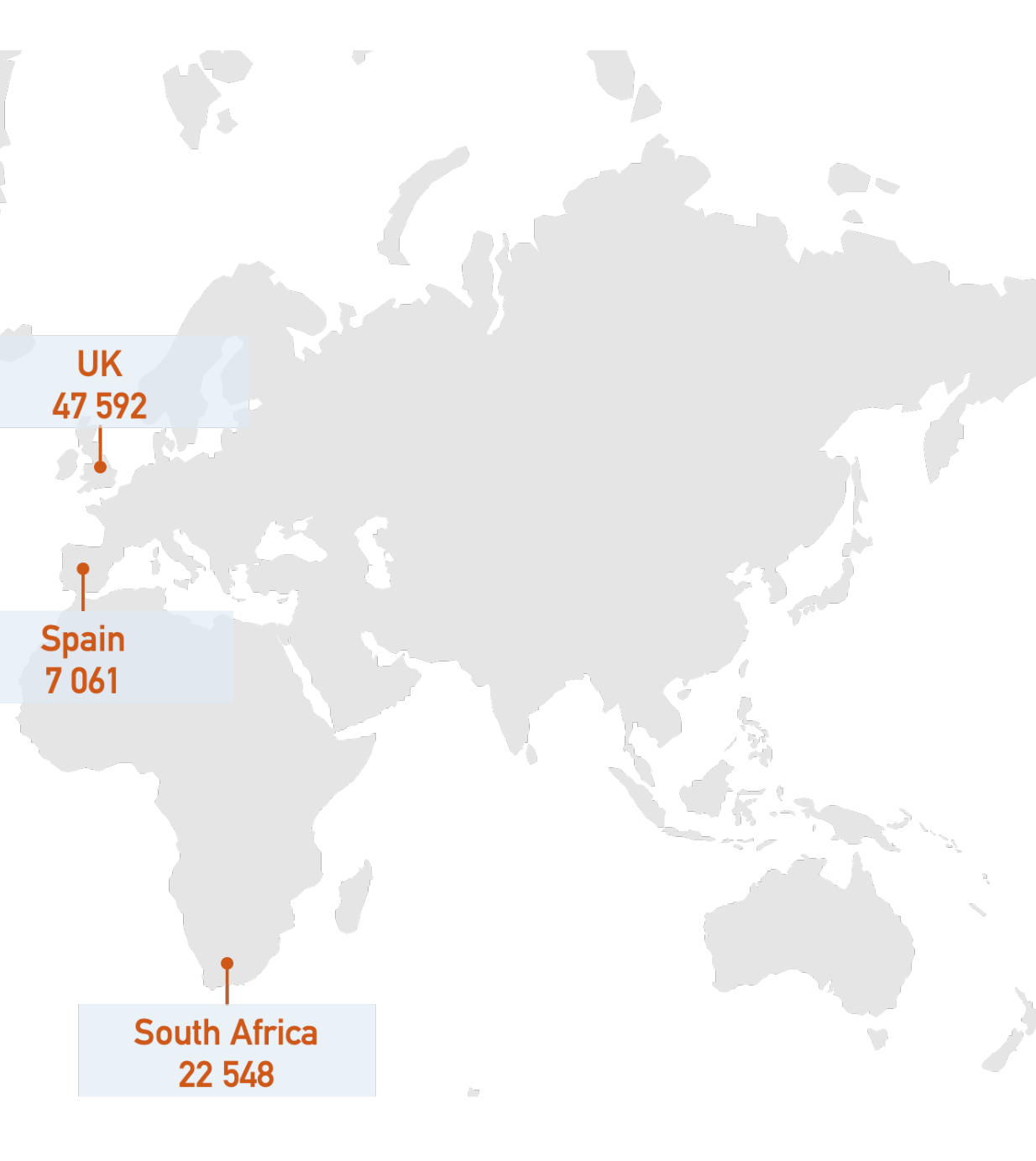

\section{CONCLUSION}

- In this meta-analysis of 11 studies with 1268676 individuals with confirmed COVID-19, we found a stronger difference in mortality by HIV status for those individuals below the age of 60 years, and over this age, HIV had an attenuated effect on mortality, suggesting that age-related mortality overshadows PLHIV related mortality.

- Further, PLHIV had increased odds of being hospitalized and needing intensive cares services, probably related to increased COVID-19 severity in PLHIV.

- A secondary analysis of the included studies suggested no difference in the prevalence of pre-existing conditions.

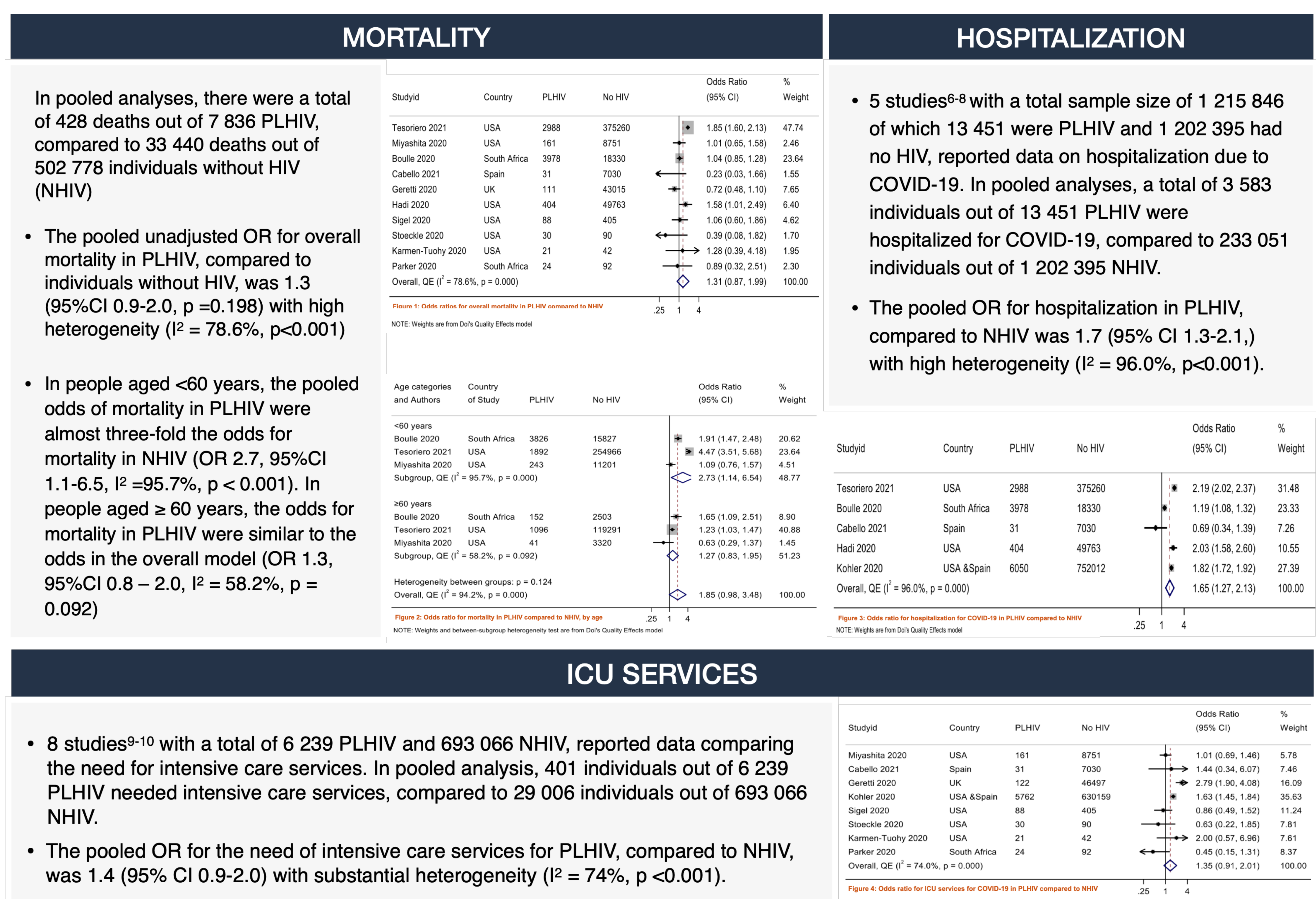

Study quality was assessed using the MethodologicAl STandard for Epidemiological Research (MASTER) scale. Data synthesis used a bias adjusted model and predefined age and geographical subgroups were analysed.

\section{MATERIALS AND METHODS \\ 1st January 2020 \\ A comprehensive search in PubMed, Cochran Library, Scopus, China Academic Journals Full Tex Database, the Database of Abstracts of Reviews of Effectiveness (DARE) and and the medRXIV and bioRxiv databases of preprints was carried out. \\ whesectional and cohort studies where participants had confirmed \\ From each study, data on numbers HIV for ach individuals without extracted.

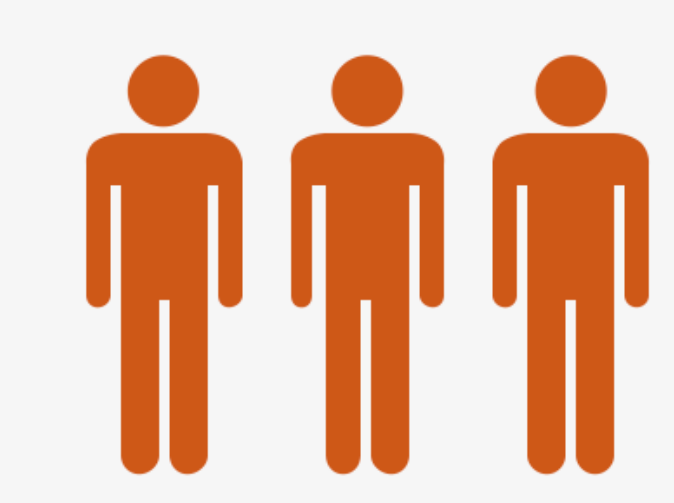

20th February 2020 